\title{
Laboreal
}

Volume $9 \mathrm{~N}^{\circ} 1$ | 2013

Varia

\section{La gestión del riesgo en la industria forestal uruguaya}

Gerenciamento de riscos no setor florestal uruguaio

La gestion du risque dans l'industrie de production de bois uruguayenne

Risks management in the Uruguayan forest industry

\section{Francisco Pucci, Soledad Nión y Fiorella Ciapessoni}

\section{(2) OpenEdition}

\section{Journals}

\section{Edición electrónica}

URL: http://journals.openedition.org/laboreal/6021

DOI: $10.4000 /$ laboreal.6021

ISSN: 1646-5237

\section{Editor}

Universidade do Porto

\section{Referencia electrónica}

Francisco Pucci, Soledad Nión y Fiorella Ciapessoni, « La gestión del riesgo en la industria forestal uruguaya », Laboreal [En línea], Volume 9 No $^{0} 1$ | 2013, Publicado el 01 julio 2013, consultado el 04 octubre 2019. URL : http://journals.openedition.org/laboreal/6021 ; DOI : 10.4000/laboreal.6021

Este documento fue generado automáticamente el 4 octubre 2019.

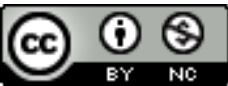

Laboreal está licenciado com uma Licença Creative Commons - Atribuição-NãoComercial 4.0 Internacional. 


\title{
La gestión del riesgo en la industria forestal uruguaya
}

\author{
Gerenciamento de riscos no setor florestal uruguaio \\ La gestion du risque dans l'industrie de production de bois uruguayenne \\ Risks management in the Uruguayan forest industry
}

Francisco Pucci, Soledad Nión y Fiorella Ciapessoni

\section{NOTA DEL EDITOR}

Manuscrito recibido en : julio/2012

Aceptado tras peritaje : junio/2013

\section{Introducción}

1 El análisis de los factores de riesgo presentes en los procesos productivos requiere, como condición previa, realizar un breve repaso sobre las concepciones teóricas que se han desarrollado recientemente en relación a la problemática del riesgo. Si bien estos desarrollos se centraron fuertemente en los riesgos ecológicos o ambientales, también pueden ser incorporados al análisis de los riesgos presentes en el mundo del trabajo. Uno de los aspectos centrales del análisis de las condiciones de trabajo es comprender las estrategias de los actores que directa o indirectamente intervienen en la construcción y cambio de las condiciones de trabajo. Cada situación de riesgo pone en evidencia una multiplicidad de actores que conforman una trama compleja, particular en cada caso, que maneja y pone en evidencia niveles de aceptabilidad del riesgo muy diferentes. Estas diferencias se deben tanto a los intereses específicos y estratégicos de los actores involucrados, como a la presencia de perspectivas, valores y códigos de interpretación de la realidad diversos y heterogéneos. Los desarrollos teóricos ligados a la gestión del riesgo permiten dar cuenta de esta complejidad y orientar la 
investigación para lograr determinar los niveles aceptables de riesgo que se establecen en una actividad productiva específica.

2 En este sentido, la noción de riesgo no es una concepción puramente técnica o científica. Hay una construcción social del riesgo que pone en juego una multiplicidad de intereses y de representaciones de parte de diferentes actores. La fragmentación y la heterogeneidad de las prácticas en esta materia provienen del hecho de que la gestión del riesgo es muy diferente según los tipos de riesgo o las regiones, movilizando estructuras de concertación más o menos institucionalizadas y referencias jurídicas diversas. Una característica de estos procesos locales de gestión de los riesgos es la poca visibilidad de las decisiones, que resultan de la acción de grupos restringidos de actores cuya actividad es poco transparente para el público. Plantear el riesgo como una construcción social permite discutir las consecuencias no deseadas producidas por el disfuncionamiento de los sistemas tanto como por la elaboración de normas o la difusión de resultados de investigación. En esta construcción social, la apreciación de los daños depende de la representación que se hacen los actores de los umbrales de peligro y de riesgo aceptable.

3 En la medida en que la gestión del riesgo está referida al manejo de la incertidumbre, la misma constituye un proceso de aprendizaje organizacional. Dado que los actores no cuentan con modelos establecidos de comportamiento a los cuales ajustarse, deben construir en la marcha los mecanismos y las actitudes para afrontar estas situaciones. Discutir la gestión del riesgo implica centrar nuestra atención en las condiciones que se requieren para que este aprendizaje pueda lograr resultados positivos en términos de minimizar los riesgos o de llegar a niveles aceptables del mismo.

4 En el caso uruguayo, la mayor parte de las empresas que implementan programas de seguridad lo hacen en el marco de una política global de certificación de calidad que incluye otros componentes, como mejora de los procesos de trabajo, mayor polivalencia de los trabajadores, etc. En este sentido, estos procesos se inscriben en una lógica racional de maximización de beneficios, en la cual la estabilidad de las políticas de seguridad está fuertemente ligada a una perspectiva de estabilidad de la empresa en el mercado, sea nacional o internacional. Pese a estas motivaciones, los estudios realizados muestran que la implementación de políticas de seguridad por parte de las empresas uruguayas es parcial y limitada $\mathrm{y}$, en los casos en que encontramos una preocupación importante por el tema de seguridad, el mismo reviste un enfoque de la gestión que no siempre permite dar cuenta de la complejidad del fenómeno (Pucci, 2004).

5 Esta situación adquiere características específicas en las nuevas actividades industriales que ha emprendido el país en los últimos años, en particular en la producción forestal. Si bien el área forestada en Uruguay se multiplicó más de 15 veces en el período 1988-2005 y la exportación de productos forestales creció 1181 \% respecto a 1987, todavía no está claro para expertos, políticos, actores sociales y laborales que la misma genere impactos sociales positivos (Riella \& Ramírez, 2007). En este sentido, los problemas de seguridad en el trabajo y las necesidades de lograr una gestión más eficiente del riesgo adquieren una relevancia central en estas nuevas actividades, en la medida que su legitimidad económica, social y ambiental aparece cuestionada desde sus inicios. El objetivo de este artículo es analizar las políticas de seguridad que se desarrollan en este sector, y en particular, las posibilidades de desarrollo de competencias organizacionales para una gestión más eficiente del riesgo, partiendo de 
la premisa de que toda política de seguridad debe inscribirse en un marco global de aprendizaje organizacional de la empresa. Este trabajo busca explorar las facilidades y las dificultades que los componentes organizacionales de las empresas forestales presentan para el desarrollo de competencias vinculadas a la gestión del riesgo.

\section{Metodología utilizada}

6 El análisis de los procesos de gestión del riesgo requiere un abordaje que de cuenta de componentes cualitativos difícilmente cuantificables, como la cultura de riesgo, las relaciones de comunicación y las reglas informales construidas frente a situaciones de incertidumbre. En función de estos objetivos, la investigación se inscribió en una mirada cualitativa de investigación, en la medida en que se requiere “...la adecuación del método a la dimensión considerada en el objeto, y ello no de manera arbitraria e intercambiable, sino con el rigor que el propio objeto demanda para que su tratamiento pueda calificarse de científico" (Beltrán, 1992, p. 44).

7 El diseño utilizado fue abierto, en la medida en que permitió la posibilidad de que se produjera información que no estuviese contemplada de antemano en el diseño (Ibáñez, 1992) y tuvo un componentes básicos de corte exploratorio-descriptivos. La metodología cualitativa distingue dos tipos de entrevistas: las no dirigidas o no estructuradas, que suelen utilizarse con fines exploratorios, y las entrevistas en profundidad o semi directivas, donde el investigador orienta el discurso lógico de una persona, por lo que cuenta con un esquema fijo de cuestiones mínimamente ordenadas $\mathrm{y}$ formuladas. En este caso, se realizaron entrevistas semi estructuradas individuales y colectivas y las mismas contemplaron los ejes analíticos del marco teórico previsto apriori pero conservando la libertad de profundizar en aspectos que fueron emergiendo de las entrevistas por ser considerados de interés para la investigación. Esta estructura mínima en las entrevistas, a través del planteamiento a priori de un guión, permitió disparar el discurso de los entrevistados (mediante un sistema de consignas) al tiempo que contempló los objetivos de la propia investigación. Tal como señala Orti (1992) mediante la técnica de entrevista se le devuelve el uso de la palabra libre a los individuos entrevistados, por lo que el investigador accede al preconsciente ideológico de los mismos, siendo posible intentar interpretar las claves de su conformación.

8 Se realizaron un total de 43 entrevistas, en el correr del año 2011, al personal de diferentes categorías laborales en 3 empresas forestales, 2 del sector pastero y 1 del sector maderero (Ver Cuadro 1). El muestreo fue teórico por tipo de puesto y etapa del proceso productivo, respetando el criterio de saturación teórica para su delimitación. 
Cuadro 1 - Entrevistas según empresa y etapa del proceso productivo

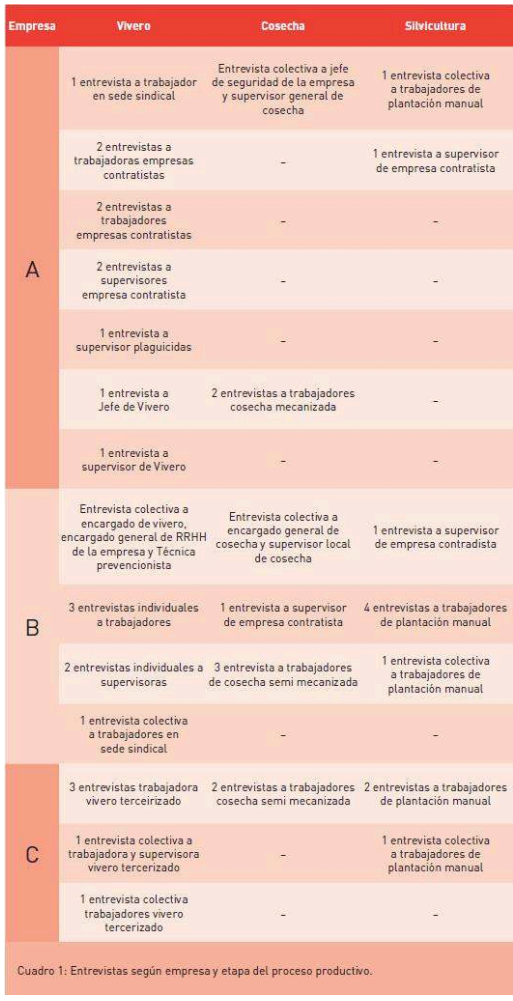

9 Las entrevistas estuvieron orientadas al relevamiento de las variables cualitativas presentes en el diseño : cultura de riesgo, rutinas laborales, relaciones de comunicación y confianza, procesos de traducción y actividad de regulación, condiciones laborales, entre otras (Ver Cuadro 2). 


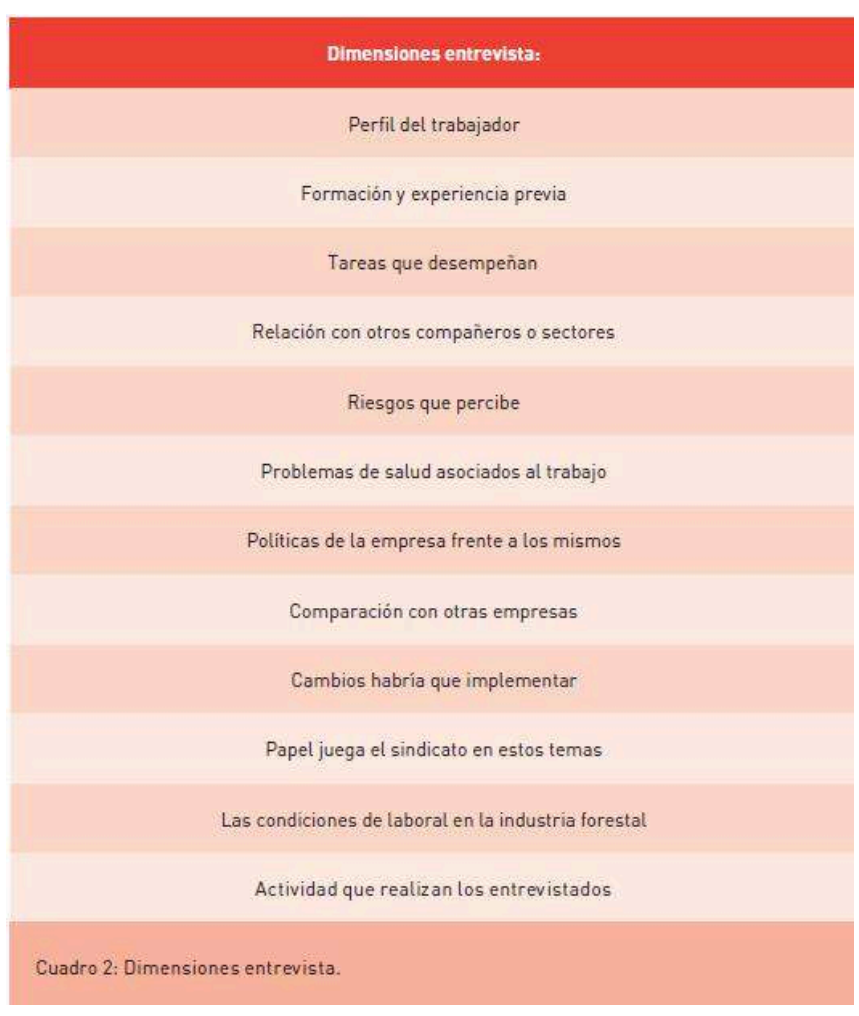

Al mismo tiempo, se realizaron dos tipos de observación : 1) observación directa de los puestos de trabajo, del proceso, de las condiciones generales de trabajo y disposición espacial y de los signos/símbolos que se encontraron en el lugar (como por ejemplo señales asociadas a dispositivos de seguridad, etc.). 2) observación indirecta, mediante el análisis de material fotográfico y audiovisual recolectado en los lugares, que permita complementar la etapa de análisis de las entrevistas una vez cerrada la etapa de recolección de la información propiamente dicha. Con este tipo de observación, se complementa las dos técnicas anteriores mediante el análisis de lo que podría ser considerado un diario de campo gráfico (García Ferrando \& Sanmartín, 1992). Ambos tipos de observación permitieron contrastar el discurso de los actores, recogido en las entrevistas, con las conductas concretas implementadas en las rutinas de trabajo, dando lugar a una nueva clase de información que tiene en cuenta la diferencia entre lo ideal (que surge en el plano discursivo) y lo real (lo observado) (García Ferrando \& Sanmartín, 1992).

\section{Organización y riesgo}

La dificultad de una organización para resolver situaciones complejas se traduce en la presencia de riesgos sistémicos, es decir, en zonas de incertidumbre permanente en el funcionamiento de la vida de la organización. Estos riesgos sistémicos expresan la incapacidad de la racionalidad para dar cuenta de la complejidad y de la incertidumbre presentes en los nuevos modelos productivos. Los análisis de Beck, (1998) Luhmann (1992) y Giddens (1994) muestran, a su vez, como las organizaciones, en la medida en que son incapaces de reducir la incertidumbre, establecen, de manera explícita o implícita, un proceso de selección de riesgos. Este proceso de selección es un proceso 
social : implica la puesta en marcha de decisiones, que pueden ser analizadas a partir de la percepción que los actores tienen de los riesgos, y de la valoración individual o institucional de los mismos. Debido a que estas percepciones y valoraciones pueden ser diferentes en los distintos grupos, estamentos profesionales o niveles jerárquicos de la organización, la selección de riesgos conlleva necesariamente una negociación, explícita o implícita, formal o informal, para definir cuáles son sus niveles aceptables. La definición del riesgo aceptable cristaliza el resultado de estos conflictos, estableciendo un compromiso más o menos estable entre los afectados por los riesgos y los decisores, que se constituye en un marco de orientación del comportamiento de los actores en los sistemas productivos.

La presencia de zonas de inseguridad en las organizaciones, tiene como consecuencia la identificación de riesgos permanentes en el funcionamiento de las mismas. Perrow (1984) mostró como determinados sistemas sociales inducen inevitablemente la presencia de riesgos, debido a su propia estructura. En cada sistema social, la presencia de estos riesgos sistémicos es un indicador de la cultura de riesgo de dicho sistema. Otra dimensión estudiada por estas perspectivas de análisis es la tendencia de las organizaciones a normalizar el desvío (Vaughan, 2001). Este proceso implica que, en las decisiones organizacionales, la información que en principio fue analizada y evaluada como un signo de peligro potencial, es, posteriormente, evaluada como aceptable y no desviada. Esta aceptación es una construcción cultural que se procesa en el grupo de trabajo, en función de las interacciones y de las representaciones del riesgo que se elaboran en el mismo.

13 También se ha destacado la situación en la cual se logran alcanzar márgenes de fiabilidad pese a la organización (Bourrier, 2001). Desde esta perspectiva, la organización es vista como un obstáculo para alcanzar los niveles de seguridad sistémica requeridas. Son las capacidades de adaptación, de anticipación y de invención de los trabajadores los que permiten sortear los escollos que la organización impone a la construcción de un modelo de seguridad sistémica.

Las corrientes de análisis organizacional se centran en el estudio de los procesos a partir de los cuales las organizaciones de alta fiabilidad logran reducir los márgenes de accidentalidad en su funcionamiento concreto. Estas organizaciones actúan sobre la base de la construcción de relaciones de confianza con el público y entre los grupos e individuos que participan de la misma. En relación a las organizaciones de alta fiabilidad, La Porte (2001) define las características que distinguen a las llamadas HRO (High Reliability Organizations) :

1. Un uso flexible de la noción de autoridad y una estructura organizativa específica para situaciones de crisis.

2. El reconocimiento activo, en todos los niveles, de las competencias, de los trabajadores y de su dedicación a la tarea.

3. Los esfuerzos constantes de formación.

4. La existencia de un sistema que recompensa la detección de errores y valoriza la trasmisión y puesta en común de las informaciones al respecto.

5. La presencia de actitudes que favorecen o frenan el cambio técnico $u$ organizacional, de acuerdo a los juicios positivos o negativos que sus efectos producen en la fiabilidad organizacional.

El eje de estas organizaciones de alta fiabilidad es lograr la confianza institucional tanto a nivel interno de la organización, como en relación a otros organismos públicos y 
privados y al público en general. Junto a la noción de confianza institucional, La Porte enfatiza en las condiciones que garantizan la constancia de esta confianza a lo largo del tiempo. En primer lugar, debe existir la voluntad política e institucional de la organización y su determinación de conservar esta confianza. En segundo lugar, la organización debe tener la capacidad de mantener intactos sus compromisos a lo largo del tiempo. Esto implica que la confianza no se origina por decreto sino que requiere un proceso de construcción. Si la confianza existe es que las normas de conducta son compartidas, y si son compartidas no aparecen distorsiones con relación a la obtención del objetivo común. La prueba de la existencia de la confianza común es que la internalización de las metas y de los medios legítimos para alcanzarlos forma parte de un acuerdo tácito. Si hay verdadera confianza, un individuo solo o un grupo aislado se van a comportan tal como se espera de ellos. Pero, siguiendo a Karpik (1996), esta internalización es el resultado de un período anterior de negociación y confrontación. Es necesaria la interacción entre el que dicta la conducta y el que obedece, y que la misma sea importante para ambos.

La construcción de relaciones de confianza implica un proceso de aprendizaje organizacional. Este concepto permite analizar la manera como la adquisición de nuevos conocimientos en las organizaciones estructuran los modos de decisión y acción. Koenig (1994) define el aprendizaje organizacional como un fenómeno colectivo de adquisición y de elaboración de conocimiento que, de manera más o menos profunda y durable, modifica tanto la gestión de las situaciones como las situaciones en sí. En esta perspectiva, se considera que los individuos evolucionan en un contexto de incertidumbre, en tanto el conjunto de elecciones posibles resulta exógeno para aquél que decide. Por el contrario, el conjunto de elecciones posibles se debe construir, debido a que la cuestión no es solamente seleccionar la mejor opción, sino que el problema es construir el conjunto de opciones posibles.

Boissières (2007), a su vez, incorpora en esta temática el concepto de robustez organizacional, como el conjunto de competencias necesarias para una gestión eficiente del riesgo. Las dos entradas privilegiadas para el análisis de los riesgos son, desde esta perspectiva, los accidentes y las vulnerabilidades de la organización. En este plano, se pueden detectar, en las organizaciones, dos tipos de regulación de las perturbaciones ordinarias : una forma de regulación orientada por la lógica securitaria, y una forma de regulación orientada por una lógica de gestión de los riesgos. Esta última es la que permite la construcción de competencias específicas para la prevención de accidentes. Su desarrollo permite enfrentar los problemas emergentes en organizaciones complejas en contextos de incertidumbre. El sustento de este proceso de aprendizaje es el desarrollo de lógicas de acción diferentes a las prevalecientes en los modelos racionales, estables y jerárquicos, cuya expresión clásica ha sido la organización taylorista-fordista del trabajo y las organizaciones.

18 En síntesis, el aprendizaje organizacional puede ser considerado como una modificación de las rutinas, de las reglas de acción y de los valores y las representaciones sobre las cuales están basadas estas reglas. Este proceso incluye la existencia de rutinas defensivas que fijan los comportamientos individuales y colectivos, aunque los mismos se traduzcan en disfuncionamientos (Argyris, 1995). En estos casos, el aprendizaje se restringe y la corrección de problemas no da lugar a una reflexión sobre los valores que dirigen las estrategias de acción. 
19 Las nuevas concepciones sobre el riesgo desarrollados hasta acá se implementan en contextos en los cuales se redefinen los principios sobre los cuales se sustenta la eficacia de la producción industrial. La performance en términos de calidad, flexibilidad y costos de la empresa, depende más, en las condiciones actuales de producción a nivel mundial, de la calidad de la organización y del nivel de comunicación de las interacciones entre los actores que de la rapidez y eficacia de las operaciones elementales (Veltz \& Zarifian, 1993). Las innovaciones organizacionales "puras" inspiradas en los modelos post-fordistas (toyotismo, justo a tiempo, etc.) adquieren tanta relevancia como la introducción de nuevas tecnologías. En estos contextos de innovación tecnológica, con presencia de tecnologías de última generación, el rediseño de los puestos de trabajo y el desarrollo de nuevas competencias se vuelve necesario para lograr una utilización más eficiente de la misma. En estos espacios de innovación y cambios organizacionales se plantea, en el campo de la seguridad, la necesidad de desarrollar competencias organizacionales específicas para lograr una gestión más eficiente del riesgo. Los procesos de aprendizaje de la gestión del riesgo se articulan con las transformaciones organizacionales inspiradas en los modelos toyotistas, y se implementan a partir de los siguientes supuestos:

a. La construcción de competencias para la gestión del riesgo implica una articulación con procesos globales de aprendizaje organizacional, que impliquen modificaciones en las rutinas de trabajo y en los comportamientos cotidianos de los actores laborales, procesos que se deben construir tomando en cuenta las diferentes perspectivas y orientaciones en juego en cado espacio concreto.

b. Este proceso de aprendizaje organizacional requiere, como condición necesaria, una fluida relación de intercambio entre agentes técnicos y agentes profanos, que genere condiciones de confianza. Los formatos institucionales más descentralizados y horizontales, en los cuales el conocimiento técnico deje de ser el referente único y absoluto, generan espacios de intercambios y negociaciones permanentes entre expertos, decisores y trabajadores.

c. La construcción de competencias para la gestión del riesgo deben partir de la base de que los actores productivos son actores reflexivos, es decir, que tienen un profundo conocimiento práctico de su actividad cotidiana, y que tienen la capacidad de dar cuenta de ese conocimiento y de hacerse responsables del mismo. Este presupuesto es fundamental para orientar la formación hacia el desarrollo de capacidades comunicativas y de autorregulación que permitan generar procesos de negociación y establecer acuerdos y compromisos ligados a la gestión del riesgo.

d. El aprendizaje organizacional se debe comprender como una experiencia de comunicación, que permita traducir las diferentes racionalidades técnicas y las diversas racionalidades prácticas que están en juego, de manera de lograr acuerdos provisorios y contingentes en torno a situaciones específicas. La transitoriedad y fragilidad de los acuerdos no debe entenderse como un componente negativo de estos procesos, sino más bien como una condición propia de los contextos de incertidumbre.

e. La construcción de competencias debe tomar en cuenta no sólo las normas y las negociaciones explícitas que se establecen entre los actores laborales, sino también los procesos de negociación implícitos que se presentan en el trabajo cotidiano, y que se cristalizan en rutinas de trabajo. El carácter implícito de estos acuerdos no se opone al concepto de reflexividad que desarrollamos en el punto anterior. Por el contrario, si bien los mismos tienen un carácter invisible y no verbalizado, los actores tienen la capacidad de racionalizar su conducta y de dar cuenta de los mismos en cualquier situación. Esta capacidad permite la comunicación de los códigos y saberes prácticos, su modificación y su adaptación a nuevas situaciones. 
f. El desarrollo de competencias debe partir del supuesto de que las orientaciones y conductas que desarrollan los actores laborales frente a los riesgos, son principalmente un producto organizacional y no la consecuencia de socializaciones externas al ámbito laboral. Si bien los trabajadores pueden ser portadores de culturas de trabajo internalizadas a través de la familia o de los diferentes espacios de socialización primaria, la actitud hacia los riesgos es la consecuencia de una determinada "cultura organizacional", que puede reforzar, mejorar o transformar estos determinantes inherentes a la socialización primaria. En definitiva, se trata de privilegiar los componentes contingentes de cada organización en relación a los determinantes estructurales o normativos de las conductas.

g. La construcción de competencias no debe estar únicamente orientada a lograr una exitosa internalización de normas de conducta o de valores asociados a la seguridad. Si bien este aspecto parece una condición necesaria para una adecuada gestión del riesgo, la manera de resolver las incertidumbres que se presentan requiere de un proceso de construcción de reglas con componentes más horizontales e implícitos, que se traduzcan en rutinas de trabajo más eficientes. Las opciones y elecciones son por lo tanto mucho menos estructuradas, más coyunturales y situacionales, sustentadas fundamentalmente sobre el conocimiento práctico de los trabajadores. Esta alternativa se apoya en modalidades de regulación más descentralizadas y autorreguladas, donde las unidades pasan a tener una gran dosis de autonomía.

\section{Antecedentes en Uruguay}

tudios realizados (Pucci, 2004) han mo la gestión del riesgo nos enfrenta a un conjunto de instituciones, organizaciones y comunidades técnicas que se encuentran en el primer peldaño de este proceso de aprendizaje. Las "culturas de riesgo" institucionales también tienen un carácter limitado y específico. A su vez, las políticas de seguridad se asocian a un modelo mecánico de evaluación de los riesgos, donde se considera que la reducción de los accidentes pasa principalmente por el cumplimiento de la normativa y la inversión económica, sin tomar en cuenta la complejidad de los procesos sociales asociados al riesgo y la irreductibilidad de los mismos. Esto se observa en la confianza que se posee en la cuantificación de los sucesos, de las probabilidades y en los esfuerzos destinados desde la organización a investigar cada accidente que se genera. Tanto los incidentes como los accidentes son conceptualizados como entidades relativamente simples, los cuales pueden ser explicados en función de causas aislables mediante el uso de la razón o racionalidad técnica. Esta concepción traduce una mirada no compleja de la causalidad, claramente visible en el énfasis que se le otorga a la información, tanto en el sistema de denuncias de incidentes, los reportes, los formularios, etc. Se parte de la base de que teniendo un aceitado sistema de información, contando con estadísticas actualizadas y realizándose los procesos de investigación adecuados, resulta posible llegar a las raíces del problema y resolverlo. Los procedimientos también reflejan una perspectiva mecánica de la gestión del riesgo, que se apoya fundamentalmente en la jerarquía vertical y en el cumplimiento de requisitos sometidos a controles externos, dejando de lado formatos más horizontales que den cuenta y se reapropien del saber y del "saber hacer" de los operarios.

21 El accidente, en este tipo de organización del trabajo, se considera como el resultado de los errores de los operarios, faltas, incumplimientos o desempeños incorrectos. En todo caso, siempre hay una falla generadora. El tratamiento del riesgo parece orientarse por 
una perspectiva individualista, en la cual el componente decisional es clave para entender la frecuencia de accidentes de trabajo. Los trabajadores, en cambio, se orientan a localizar los accidentes en errores de organización del trabajo, de supervisión o de incumplimiento de normas de seguridad. Su perspectiva se asocia más al modelo del error humano, lo que implica no tomar en cuenta los componentes organizacionales para explicar la accidentalidad. En ambas perspectivas, los accidentes siempre son vistos a la luz de un error cometido y no como el producto de la actividad organizacional entendida como un sistema complejo. Si bien estos componentes muchas veces están presentes, los accidentes no son analizados en el marco de la complejidad creciente de los sistemas productivos, que genera zonas de riesgo sistémico, consecuencias no deseadas y efectos perversos de diferente tipo, lo que obliga a trasladar la mirada desde la responsabilidad individual a la responsabilidad organizacional o colectiva.

Una gestión del riesgo más eficiente debe tomar en cuenta no sólo las normas y las negociaciones explícitas que se establecen entre los actores laborales, sino también los procesos de negociación implícitos que se presentan en el trabajo cotidiano, y que se cristalizan en rutinas de trabajo. El carácter implícito de estos acuerdos no se opone al concepto de reflexividad mencionado más arriba. Por el contrario, si bien los mismos tienen un carácter invisible y no verbalizado, los actores tienen la capacidad de racionalizar su conducta y de dar cuenta de los mismos en cualquier situación. Esta capacidad permite la comunicación de los códigos y saberes prácticos, su modificación y su adaptación a nuevas situaciones.

23 Sin embargo, en los casos estudiados, la gestión del riesgo no parece articularse con procesos globales de aprendizaje organizacional, que impliquen modificaciones en las rutinas de trabajo y en los comportamientos cotidianos de los actores laborales, procesos que se deben construir tomando en cuenta las diferentes perspectivas y orientaciones en juego en cado espacio concreto. Este proceso de aprendizaje organizacional requiere, como condición necesaria, una fluida relación de intercambio entre agentes técnicos y agentes profanos, que genere condiciones de confianza. La jerarquía y la centralización de las decisiones deben ser sustituidas por formatos institucionales más descentralizados y horizontales, en los cuales el conocimiento técnico deje de ser el referente único y absoluto, generando espacios de intercambios y negociaciones permanentes entre expertos, decisores y trabajadores. Esta dimensión no parece presente en la mayoría de las empresas uruguayas.

La gestión del riesgo está orientada, en el mejor de los casos, a lograr una exitosa internalización de normas de conducta o de valores asociados a la seguridad. Si bien este aspecto parece una condición necesaria, la manera de resolver las incertidumbres que se presentan requiere de un proceso de construcción de reglas con componentes más horizontales e implícitos, que se traduzcan en rutinas de trabajo más eficientes. Las opciones y elecciones son por lo tanto mucho menos estructuradas, más coyunturales y situacionales, sustentadas fundamentalmente sobre el conocimiento práctico de los trabajadores. Esta alternativa se apoya en modalidades de regulación más descentralizadas y autorreguladas, donde las unidades pasan a tener una gran dosis de autonomía. La recuperación y comunicación del saber práctico de los trabajadores y de su capacidad de generar, de manera colectiva, reglas de conducta adecuadas a los contextos de incertidumbre, es una condición central en los procesos de gestión del riesgo. Esto implica pensar los mismos desde modelos más complejos que aseguren el 
aprendizaje permanente y la construcción cotidiana de comportamientos y reglas que articulen el saber técnico y el saber profano.

Estos son los antecedentes a partir de los cuales abordaremos el estudio de la gestión del riesgo en la actividad forestal. En este sector, como veremos más adelante, confluyen lógicas de trabajo típicas del mundo rural con componentes modernos propios de la actividad industrial, signada por fuertes inversiones tecnológicas y por formas de gestión del riesgo que se inspiran en las perspectivas organizacionales de las HRO desarrolladas anteriormente. Estas formas de gestión asociadas a las nuevas formas de organización del trabajo de inspiración toyotista se despliegan en contextos de relaciones laborales precarias y con fuertes segmentaciones en los procesos de trabajo, los que establecen importantes límites y condiciones para una eficiente implementación de las mismas.

\section{Modelos y configuraciones productivas}

Para poder comparar las características de las empresas forestales uruguayas con sus similares de América Latina, resulta útil tomar el concepto de configuraciones sociotécnicas de trabajo desarrollado por De la Garza Toledo (2010). Este autor discute la pertinencia de la categoría "modelo productivo" para explicar las características de buena parte de las organizaciones productivas latinoamericanas. Las variables que generalmente se incluyen para construir este concepto son la estrategia de negocios de la organización, su política productiva, la relación salarial y las formas de gestión de la mano de obra. Además de dejar de lado algunas dimensiones como el nivel de desarrollo tecnológico y las culturas laborales y gerenciales, el concepto de modelo productivo tiene, en opinión del autor, un fuerte rasgo racional y funcionalista, que no deja espacio para el estudio de las múltiples contradicciones que se desarrollan en el marco de un proceso productivo determinado. De esta manera, categorías como taylorismo-fordismo, toyotismo, modelos post-fordistas, etc., no dan cuenta de la diversidad de situaciones de las empresas en América Latina; su ámbito de aplicación se restringe a grandes empresas del mundo desarrollado, en general de la rama automotriz

Otros autores han explicado los procesos de reestructuración productiva a través del concepto de "hibridación" (Novick, Yoguel, Catalano, \& Albornoz, 2002). Esta perspectiva parte de la base de que existe una interacción compleja entre los modelos de organización del trabajo, las decisiones estratégicas de los núcleos empresariales o de las autoridades jerárquicas y los contextos nacionales e institucionales en los cuales se implementan estos procesos. El resultado es que, en América Latina, las formas de organización del trabajo tienden a asumir formas "híbridas", en las cuales se yuxtaponen y se entremezclan componentes de diferentes modelos, sobre la base de la transformación o redefinición de los equivalentes funcionales del modelo de origen. En este plano, muchas de las nuevas formas de organización del trabajo implementadas en nuestros países no asumen la lógica integral de los nuevos modelos productivos, sino aspectos parciales y fragmentarios de los mismos. Otro componente importante de estos procesos es que, en numerosas ocasiones, el modelo original de trabajo tiene una fuerza emblemática que permea todas las futuras transformaciones que se dan en la organización de la empresa o del servicio. El problema de esta perspectiva es que sigue atada a las realidades específicas de un sector productivo y no abre la posibilidad para 
la construcción de categorías más pertinentes para entender las complejidades de la organizaciones productivas ; un modelo denominado híbrido puede ser, en realidad, un nuevo modelo de organización del trabajo no teorizado anteriormente, en el cual las dimensiones predominantes sean diferentes a las de los modelos productivos ya establecidos.

Para superar estas dificultades, De la Garza Toledo propone el concepto de configuración sociotécnica del proceso de trabajo, que incluye el nivel de desarrollo tecnológico, la forma de gestión de la mano de obra y de organización del trabajo, las relaciones laborales, el perfil de la mano de obra y las culturas de trabajo, dimensiones que pueden tener contradicciones entre sí y haber alcanzado diferentes niveles de estructuración. Mientras el concepto de modelo de producción se asocia a la idea de la coherencia de un sistema que no tienen contradicciones, el concepto de configuración productiva acepta la posibilidad de contradicción entre algunos de sus elementos, variando la extensión y profundidad de las mismas para cada caso concreto. También puede implicar que las relaciones entre sus partes tienen diferentes niveles de dureza o laxitud, dependiendo de los contextos en los cuales emerge la misma. Por último, el concepto de configuración productiva permite superar las miradas estructuralistas, que explican la emergencia de los diferentes modelos productivos debido a las coerciones impuestas por la lógica racional de la economía, destacando en cambio la acción de los actores laborales y empresariales como componentes constitutivos de estas mismas configuraciones. De esta manera, hablar de configuraciones productivas resulta más útil para dar cuenta de la complejidad de las realidades productivas de América Latina, marcadas por numerosas asincronías y diferentes niveles de desarrollo, y por la acción del Estado, de las estrategias empresariales y del papel jugado por los sindicatos.

Para México, De la Garza Toledo señala que las nuevas formas de organización del trabajo, inspiradas en el modelo toyotista, tuvieron que adaptarse, como ocurrió anteriormente con el taylorismo, a las condiciones locales de cada país, en términos de niveles de inversión, composición de la mano de obra, relaciones laborales y culturas de trabajo. Esta nacionalización del toyotismo, que pasó por las decisiones de los actores empresariales, de los sindicatos y del Estado, terminó conformando una configuración sociotécnica que De la Garza denomina "toyotismo precario", como forma dominante de la reestructuración productiva de México. Esta configuración implicó la aplicación parcial de algunos de los componentes del modelo toyotista, como el justo a tiempo y el control total de la calidad, la continuación de la segmentación entre trabajadores técnicos y operadores de planta, el achatamiento o inexistencia de carreras profesionales, la flexibilidad salarial combinada con incentivos por productividad y la persistencia de bajos niveles salariales.

30 Este tipo de configuración productiva tiene puntos de contacto con las formas organizativas de las empresas forestales uruguayas, que son el sustento de las políticas de gestión del riesgo que se implementan en las mismas. El sector forestal, en Uruguay, ha experimentando en los últimos años un proceso de modernización de su actividad productiva, a través de la implantación, en diferentes regiones del país, de empresas nacionales o extranjeras, que realizaron importantes inversiones en infraestructura y tecnología. Esta modernización tuvo como consecuencia un creciente proceso de formalización de la mano de obra contratada para trabajar en el sector, mejorando notoriamente las condiciones de empleo de los trabajadores de las regiones en las 
cuales las empresas se instalaron, los cuales se desempeñaban en actividades rurales de baja calificación y en condiciones precarias desde todo punto de vista.

\section{La producción forestal}

El perfil de los trabajadores que se incorporan a la actividad forestal se diferencia del clásico trabajador de las estancias ganaderas del Uruguay. La mayoría procede de zonas urbanas y tiene una fuerte presencia de jóvenes con bajos niveles de educación formal. Una novedad de este tipo de actividad es la incorporación de la mujer al trabajo forestal, en particular en los procesos de trabajo de plantación de los viveros, en los cuales su manualidad más fina la hace preferible en relación al sexo masculino.

La modernización del sector en términos tecnológicos y de organización del trabajo cambian las características típicas del trabajo rural, sustentado en la fuerza física y en la resistencia corporal, para aproximarlo a las modalidades del trabajo industrial. Este cambio tiene como consecuencia el aumento de las exigencias de capacitación y formación de la mano de obra, que debe desarrollar capacidades y habilidades para manejar tecnologías y procesos de trabajo más calificados. Sin embargo, pese a estos importantes cambios que acompañaron la modernización del sector, la actividad forestal mantiene dos características históricas : la estacionalidad y la tercerización del proceso de trabajo.

La estacionalidad de la producción forestal es una condición inherente a un tipo de actividad en la cual la dinámica y evolución de los factores naturales juegan un rol importante, a lo cual se le puede agregar como factor complementario las oscilaciones de la demanda de productos forestales. Esta característica de la producción forestal deriva en las modalidades de contratación de la mano de obra. Si bien la actividad forestal implicó un fuerte proceso de formalización de las condiciones de empleo, la figura predominante sigue siendo la del trabajador jornalero, que cobra su salario de acuerdo a la cantidad de días que trabaja, y que está sujeto a los vaivenes de la demanda de trabajo inherente a la estacionalidad de la producción. Junto a este trabajador jornalero, que ha constituido la modalidad histórica de contratación de la actividad forestal, se conforma un grupo de trabajadores estables, que cobran mes a mes con independencia de los ritmos de producción, y que desarrolla actividades de mayor nivel de responsabilidad para la empresa.

La estacionalidad de la producción y la necesidad de contar con trabajadores jornaleros cuando las necesidades de producción lo determinan, deriva en la segunda característica significativa de la actividad forestal uruguaya : la fuerte tercerización de sus procesos de trabajo, desde los cuales emerge una figura típica del mundo forestal: el contratista. Los diferentes componentes del proceso de trabajo de la producción forestal están a cargo de diferentes contratistas, que se ocupan de proveer la mano de obra y, en algunos casos, los implementos necesarios para el desarrollo de la misma. El proceso de trabajo forestal se compone, en función de la estacionalidad de la producción, de múltiples cuadrillas pequeñas de trabajadores jornaleros, dependientes de un contratista quien, a su vez, es contratado por la empresa forestal en la cual se desempeñan trabajadores estables que realizan tareas de supervisión, coordinación o dirección. importantes impactos en los hábitos de trabajo arraigados en los trabajadores, en la 
medida en que, para alcanzar niveles salariales relativamente decorosos, deben desarrollar su trabajo en ritmos y condiciones diferentes a las que estaban acostumbrados. La productividad pasa a transformarse en una condición de empleo, en la medida en que establece un silencioso proceso de selección de la fuerza de trabajo, en función de las capacidades de los trabajadores para alcanzar los niveles de producción exigidos. La incorporación de la productividad también conlleva un proceso de segmentación de la fuerza de trabajo, en la medida en que los salarios se distribuyen de acuerdo a los niveles de producción individuales, generando condiciones de competencia al interior de la fuerza de trabajo.

De todas maneras, si bien mantienen algunas de las características históricas de la actividad forestal, los procesos de modernización experimentados en el sector forestal uruguayo generaron condiciones de trabajo muy superiores, desde todo punto de vista, a las que existían previamente. La implementación de políticas de seguridad en el trabajo y de capacitación de mano de obra para mejorar los niveles técnicos y de seguridad de la producción constituye un cambio cualitativo en las condiciones de trabajo del sector, que no tiene antecedentes en las formas de producción anteriores.

\section{Políticas de seguridad y gestión del riesgo}

37 Las políticas de gestión del riesgo implementadas por las empresas forestales se inspiran en los mismos principios de las organizaciones de alta fiabilidad (HRO) estudiadas por La Porte (2001) superando los modelos de conquista de seguridad basados en la premisa de que la suma de medidas parciales de seguridad, como el desarrollo de normativas, la implementación del uso de elementos de seguridad personal y la capacitación aseguran por sí mismos la seguridad total del sistema. Las políticas de seguridad implementados por las empresa forestales, en cambio, retoman los lineamientos asociados a las modelos probabilísticos de gestión del riesgo, que consideran el accidente una probabilidad siempre presente en los procesos de trabajo, por lo que no existen situaciones de riesgo cero.

\footnotetext{
“Toda, toda tarea tiene riesgo, nosotros tenemos un, una serie de tareas que son las normales, los procedimientos de trabajo generales, no ?...Procedimientos escritos..para ...todas las tareas están desarrolladas, de alguna manera escritas como tienen que ...que realizarse, este...están los procedimientos, están los instructivos de trabajo para cada una...de los puestos de trabajo, o de las tareas allí, que allí están considerados, están evaluados los riesgos, tanto los laborales como..los.ambientales. Eso es una primera guía. Luego tenés, eh...tareas no rutinarias que pueden surgir en algún momento, se hace una evaluación especial para esa tarea no rutinaria que se evalúa con la gente de PRL, Prevención de Riesgo Laboral." (Extraído de entrevista a Jerarca empresa forestal)
}

Las políticas de seguridad analizadas también se inspiran, de manera más o menos consciente, en lo que Boissières (2007) denomina modelos sustentados en el robustecimiento de los dispositivos de prevención de riesgos, a través del estudio de las perturbaciones organizacionales consideradas como normales. Esta perspectiva se expresa, en el caso de las empresas forestales analizadas, en el estudio pormenorizado que los técnicos en seguridad realizan de los incidentes menores o de los desvíos de las 
conductas prescriptas. De esta manera, se anticipan los accidentes mayores corrigiendo los desvíos menores que se producen a diario en los procesos de trabajo.

Estas políticas implicaron el desarrollo de procesos de capacitación en las tareas más riesgosas de la producción forestal, como el manejo de las motosierras y la actividad de cosecha en general, para las cuales se comenzó a exigir credenciales de formación que garantizaran el conocimiento del trabajador en los aspectos técnicos y normativos requeridos para desempeñar su tarea en las condiciones de seguridad adecuadas. Estas políticas tuvieron impactos positivos en términos de disminución de accidentes de trabajo en las empresas forestales más modernizadas.

Otro aspecto asociado a la implementación de políticas de seguridad en el sector fue la generalización de la utilización de elementos de protección personal de acuerdo a las diferentes tareas desempeñadas por los trabajadores. Las empresas forestales asumen la responsabilidad de proveer a los trabajadores de los elementos de protección adecuados, además de reponerlos cuando se deterioran. También se responsabilizan de brindar a los trabajadores la ropa de trabajo adecuada, la que constituye también un elemento de protección personal en el contexto de una actividad que se desarrolla al aire libre, sometida a los impactos de los elementos naturales. Esta política de suministro de elementos de protección personal se acompaña de políticas de capacitación que apuntan a internalizar la necesidad de la correcta utilización de estos elementos por parte de los trabajadores.

41 La perspectiva de la gestión del riesgo inherente a las políticas de seguridad implementadas por las empresas forestales asumen el hecho de que los que participan del proceso de trabajo son agentes reflexivos, con capacidad para incorporar conocimientos técnicos y de tomar decisiones en contextos inciertos. En este sentido, la incorporación de los saberes técnicos a la experiencia práctica de los trabajadores, permite que los mismos desarrollen competencias específicas vinculadas a la gestión del riesgo, generando también una conciencia crítica con respecto a las decisiones que toma la empresa en la temática. El desarrollo de competencias para la gestión del riesgo enriquece el proceso de negociación de los umbrales aceptables del riesgo de la organización, en la medida en que los actores que participan en la misma tienen una elaborada cultura de riesgo y han desarrollado capacidades para la gestión del mismo.

Estos dispositivos generan las posibilidades de conformar culturas de riesgo, a nivel organizacional, altamente elaboradas, en las que se supera la perspectiva de naturalización del riesgo, muy común en los procesos de trabajo del mundo rural, y se la sustituye por percepciones y valoraciones del riesgo que parten de la premisa del carácter social del mismo, asociado a decisiones humanas, lo que lo diferencia claramente del peligro.

\footnotetext{
“Aparte por el tema mismo de los productos químicos, son productos químicos vos en el momento andás...recién aplicado te metés a trabajar, en el momento nada sentís...lo que sea, pero eso se te va acumulando en el cuerpo, después con los años empezás a tener los síntomas de...y cosas así no... sabemos que...de repente en un futuro no sé cómo vamos a estar..." (Fragmento de entrevista a Trabajadores empresa forestal)
} organización, de normas y valores comunes, de carácter contextual y provisorio, sobre 
los comportamientos y actitudes de todos los estamentos laborales, en relación al riesgo.

\section{La negociación de los umbrales aceptables de riesgo}

as Comisiones de seguridad de la empresa, o de las demandas que establecen en su acción reivindicativa. Estos procesos de negociación cuentan con el respaldo de la normativa aprobada a partir del año 2005 en Uruguay en materia de seguridad, pero se desarrolla en un contexto de relaciones laborales marcado por la debilidad de las organizaciones sindicales de la rama forestal, reflejo de la falta de tradición sindical que históricamente caracterizó al mundo del trabajo rural.

\footnotetext{
"En la forestación en estos años se han ido procesando algunas cuestiones que han ido regularizándose, un sector que tuvo mucha informalidad, mucha desregulación laboral, más allá de que hubo algunas herramientas en las cuales el sindicato ha participado, como es el Decreto 372/99, fundamental para el trabajo con la madera, porque regula todas las condiciones de salud y seguridad laboral, las condiciones de trabajo en el monte, de manera de regular el trabajo de los obreros forestales." (Dirigente sindical forestal)
}

47 Las condiciones de contratación de la mano de obra, pese al aumento de los niveles de formalización del trabajo, establecen limitaciones importantes al desarrollo de la acción sindical, dados los altos niveles de vulnerabilidad que las mismas establecen para los trabajadores rurales. La fuerte descentralización del proceso de trabajo, agrega otro factor negativo al desarrollo de la acción sindical, dada la alta fragmentación del proceso de trabajo, sustentado en cuadrillas organizadas por contratistas, que mantienen lógicas autónomas de contratación. 

incorporados al sector, la dependencia directa de los trabajadores de la figura del contratista, la permanente amenaza de desempleo típico del carácter cíclico de este tipo de producción, y las dificultades para construir liderazgos sólidos, contribuyen a mantener la debilidad del sindicalismo en el sector. La estructura descentralizada de la producción tiene su correlato en una estructura sindical que también es altamente descentralizada, lo que dificulta el desarrollo de acciones colectivas. En este contexto, la negociación de los umbrales aceptables de riesgo no siempre se expresa en acciones sindicales. En muchos casos, sus formas de expresión son más silenciosas, y se remiten a acciones cotidianas puntuales que establecen los límites entre lo que es aceptable y lo que no lo es en materia de riesgos laborales. acciones prácticas que emprenden los trabajadores, como negarse a trabajar cuando las condiciones de trabajo son malas, o negarse a tomar el agua supuestamente potable que brinda la empresa. Esta negociación también se procesa a través del desarrollo de rutinas autónomas, como procurarse el agua necesaria para el trabajo o sacarse los guantes para manejar mejor las tijeras y evitar riesgos de corte. Estas acciones muchas veces se mantienen en estado invisible, sin ser capitalizadas por la organización para mejorar los niveles de gestión del riesgo.

Más allá de los aspectos altamente positivos que acompañan el desarrollo de las políticas de seguridad y de la notoria mejora relativa en relación a otros contextos y a otros períodos históricos de la producción forestal uruguaya, la implementación de estas políticas debe enfrentar dos grandes desafíos: las condiciones de empleo del sector y las formas de gestión de la mano de obra. La zafralidad del trabajo y la presencia de la modalidad de contratación por jornal, conspiran con procesos de formación de la fuerza de trabajo en el largo plazo, que requieren condiciones de estabilidad que no están presentes en el trabajo forestal. Este desafío se ve atenuado por el hecho de que, si bien las condiciones de contratación son inestables y dependen de las demandas de producción, la fuerza de trabajo se compone de los mismos individuos que rotan de empresa en empresa de acuerdo a las ofertas de trabajo demandadas.

\footnotetext{
"Siempre el jornal. Si la máquina bueno, trabaja bien, o trabaja, como quien dice ; y hace madera, hace 10 palos, se les pagan 10 palos. $O$ sea se les paga el jornal más los 10 palos. Si la máquina trabaja normal de vuelta, no le pasa nada, se les paga el jornal, y si en vez de hacer 10, hacen 100 , se les paga 100. Así, el jornal siempre lo ganan. Lo que es variable es la producción diaria." (Supervisor cosecha)
}

51 La introducción de la productividad también constituye un desafío importante para la implementación de políticas de seguridad. La aceleración de los ritmos de realización de las tareas tiende a ser un factor que impide que el trabajador tome las debidas precauciones en su trabajo, o que no cumpla de manera adecuada con las normas vinculadas a la ejecución de las tareas.

"Ellos tienen una producción básica que es para, para ganarse el jornal tienen una producción, no ?, y a partir de ahí, todo lo que hacen por encima eso, ellos ganan un dinero extra,...hay gente que le interesa, hay gente que No le interesa." (Fragmento de entrevista a Supervisora vivero) 
tendencia a descuidar los procesos a través de los cuales se alcanzan estos resultados, lo que aumenta los riesgos de accidentes o de lesiones en los trabajadores. Por otra parte, la introducción de la productividad estimula la competencia entre los trabajadores, lo que inhibe el desarrollo de relaciones de comunicación y de apoyo mutuo entre los mismos. Esta fisura en las relaciones de cooperación conspira contra el desarrollo de un conocimiento colectivo que permita enfrentar mejor las situaciones de riesgo inherentes a la actividad forestal. También es un factor que inhibe el desarrollo de acciones o reacciones de apoyo entre los trabajadores en caso de dificultades o riesgos en la actividad.

El tercer desafío importante para el desarrollo de políticas de seguridad en la actividad forestal es la fuerte tercerización del proceso de trabajo que caracteriza este tipo de producción, debido a la estacionalidad de la misma.

\begin{abstract}
"Muchas veces tú tenés contratistas que son empresarios grandes que tienen cien, doscientas, trescientas personas, pero otras veces tenés contratistas que tienen cuatro personas, entonces, son trabajadores y cuando son trabajadores la gestión administrativa no va alineada con su gestión operativa, entonces, muchas veces hay que ayudarlos a que, de alguna forma sepan que, que tienen que cumplir con determinadas normas y que, y que nosotros sí estamos dispuestos a ayudarlos pero también si no las cumplen, indudablemente que tenemos que tener algunos mecanismos para, para corregir esas situaciones de desvío." (Fragmento de entrevista a Jerarca empresa forestal)
\end{abstract}

Si bien la empresa contratante es la que fija las condiciones de trabajo y establece las normativas generales de las políticas de seguridad, su traducción efectiva se hace a través de los múltiples contratistas de los cuales dependen las diferentes cuadrillas de trabajadores que componen el proceso de trabajo. Esta complejidad del proceso productivo pone de relieve la necesidad de establecer espacios de comunicación entre la empresa contratante y los diferentes contratistas, cada uno de los cuales es portador de un estilo de trabajo específico. La implementación de las políticas de seguridad obliga a la empresa forestal a establecer múltiples coordinaciones y relaciones de intercambio entre los encargados de definir los lineamientos de las políticas de seguridad y los actores más relevantes del proceso de trabajo, de manera de asegurar la continuidad y eficacia de las mismas.

La complejidad del proceso de trabajo forestal pone de relieve la necesidad de generar procesos de gestión de la fuerza de trabajo que acompañen y complementen los esfuerzos realizados en términos de seguridad. En este sentido, la modernización productiva no parece acompañarse, en las empresas analizadas, de una modernización de la gestión de recursos humanos. Estas formas de gestión están marcadas por la persistencia de relaciones de tipo tradicional, basadas en criterios particularistas y relaciones con fuerte presencia de vínculos familiares y parentales. Las relaciones jerárquicas se sustentan en formas de paternalismo inherentes al mundo rural, lo mismo que las relaciones de cooperación entre los trabajadores, las que están fuertemente marcadas por relaciones de proximidad familiar o regional. Este tipo de vínculo común en el mundo rural o en pequeñas zonas urbanas, se inserta en procesos de trabajo modernizados, generando un espacio de relaciones sociales específico, que no es similar al de la actividad rural clásica pero tampoco al de la actividad industrial. 

por la fuerte tercerización del proceso productivo, lo que refuerza la construcción de realidades sociales en las cuales se intensifican las relaciones internas de cada grupo o cuadrilla pero se construyen espacios heterogéneos en términos de vínculos personales en el marco global del proceso de trabajo. Esta fuerte heterogeneidad del proceso de trabajo en términos de relaciones sociales, constituye una dificultad importante en la construcción de relaciones de comunicación más fluidas entre los que implementan las políticas de seguridad y aquellos que las ejecutan. También constituye una dificultad para el desarrollo de políticas generales basadas en criterios generales y universales, que se deben implementar en espacios fuertemente marcados por vínculos personales y particulares. De todas maneras, estas dificultades no implican que las políticas de seguridad no sean eficaces: más bien lo que hacen es imponer formas de gestión específicas que contemplen la singularidad de los procesos de trabajo de la producción forestal. relaciones de confianza entre los trabajadores y la dirección, de manera de fortalecer las capacidades de la organización para detectar posibles riesgos o para evitar riesgos ya presentes. Estas formas de confianza se construyen entre actores con formaciones y racionalidades diferentes, los cuales, a través de los intercambios y la puesta en marcha de dispositivos de promesa y de juicio, obtienen los comportamientos que esperan uno del otro. En el caso de las empresas forestales analizadas, las formas de confianza que se desarrollan se basan en criterios adscriptos de tipo tradicional como la pertenencia a la misma familia o a la misma zona geográfica. Estas formas de confianza refuerzan las relaciones inter-personales en el seno de las cuadrillas de trabajadores que tienen un mismo origen y son reclutados por el mismo contratista, pero no generan formas de confianza entre las diferentes cuadrillas o en relación al funcionamiento de la organización como conjunto.

58 La fuerte presencia de dimensiones familiares y de vecindad en las relaciones sociales que se establecen en los procesos de trabajo de la industria forestal, también constituye un factor de integración social que puede ser aprovechado para mejorar la eficacia de las políticas de seguridad. En la medida en que reducir los niveles de riesgo de una actividad implica generar procesos de movilización de la fuerza de trabajo que generen un involucramiento de los trabajadores con los objetivos de las políticas de seguridad, la conformación de espacios con fuertes lazos de identidad grupal puede ser funcional a estos procesos.

Los modelos de gestión con componentes tradicionales coexisten con el desarrollo de políticas universales en materia de seguridad en el trabajo, a través de dispositivos de autoridad cuya legitimidad se construye en el marco de las relaciones cara a cara entre el jerarca y sus subordinados. Esta coexistencia de procesos de vocación universal con relaciones particularistas hace recaer en la relación que los contratistas tienen con sus trabajadores las mayores o menores posibilidades de éxito de los procesos de gestión del riesgo en las empresas forestales. En este sentido, la atribución de las responsabilidades en caso de accidentes también tiende a descentralizarse, aunque la gestión global del proceso de trabajo se realiza a nivel central. Las tensiones entre la fijación de criterios centrales en las políticas de seguridad y la descentralización de las responsabilidades constituye otro desafío importante en materia de gestión del riesgo del mundo forestal. 
60 Las políticas de capacitación de la mano de obra que forman parte del desarrollo de los procesos de mejora de la seguridad laboral, también se ven influidas por las modalidades tradicionales de gestión de la fuerza de trabajo. En algunos casos se mantienen modelos de aprendizaje basados en la idea de que se aprende realizando la tarea y en contacto con otros trabajadores, lo que implica que la incorporación de criterios de seguridad en el trabajo depende de los vínculos del trabajador con su entorno y no del impacto de espacios formales de capacitación. Las políticas de capacitación tampoco son aprovechadas por las empresas de manera óptima, en la medida en que la formación que adquieren los trabajadores no se traduce en modificaciones de las formas de organización del trabajo que permitan expandir los conocimientos adquiridos al conjunto de la empresa.

\section{Conclusiones}

61 La inclusión de las empresas forestales dentro de la categoría de "toyotismo precario" definida por De la Garza permite entender las múltiples contradicciones de estas formas de organización del trabajo. Por un lado, siguiendo el modelo toyotista clásico, las empresas forestales se han preocupado por generar procesos de participación de la mano de obra en temas de calidad y seguridad y se han desarrollado políticas de capacitación y de formación del personal inexistentes anteriormente en el sector. Esta matriz toyotista se combina con una estructura organizativa fuertemente tercerizada, que mantiene fuertes diferencias entre trabajadores estables y de producción y que genera pocos espacios para el desarrollo de carreras laborales. La misma se inserta en un medio donde predomina mano de obra poco calificada y con escasa tradición sindical, con relaciones salariales caracterizadas por una fuerte rotación del personal y por incentivos por productividad, que entran en contradicción con las políticas de capacitación y movilización de la fuerza de trabajo típicos de los modelos clásicos toyotistas.

62 En este sentido, como plantea De la Garza Toledo, el toyotismo, como el taylorismo, aunque mejore la motivación de los trabajadores, se sustenta en la intensificación del trabajo, lo que marca también sus límites físicos y sociales. Los límites físicos se visualizan en los límites de la capacidad física para alcanzar los niveles de productividad exigidos por las empresas. Los límites sociales aparecen en la insatisfacción crónica de los trabajadores, la alta rotación de mano de obra y la ausencia de carreras laborales que permitan que el trabajador se identifique con los objetivos de la empresa, pese a todas las estrategias de motivación que se implementan.

Alamberti (2012) sostiene que la idea de un modelo único de seguridad aplicable a todos los sistemas es inocente. La seguridad es una construcción social que se adapta a la demanda. Existen varias respuestas posibles que generan distintos modelos de seguridad (resiliente, HRO, ultraseguro) que poseen su propia lógica de seguridad, sus ventajas y sus límites. Estos modelos se diferencian unos de otros en función de la balanza que muestren entre el nivel de seguridad y el de adaptabilidad. Esto implica que los diferentes modelos de seguridad generan también diferentes culturas de seguridad (Simard, 1998). La adaptación a las condiciones específicas de producción es una de las causas de la tendencia a la normalización del desvío en términos de seguridad laboral. Como lo indica Boissières (2007), este fenómeno se presenta cuando la transgresión de reglas importantes de seguridad no sólo es ampliamente conocida 
sino además tolerada y aceptada por los pares y la jerarquía. La fuerte tensión entre las presiones económicas y las exigencias de seguridad favorece este proceso, en el cual los actores administran esta tensión desviándose habitualmente de ciertas normas de seguridad, porque estiman que su aplicación rigurosa es negativa para el desempeño económico de la empresa o para su condición laboral específica.

Las políticas de gestión del riesgo implementadas en las empresas forestales analizadas, si bien mejoraron notoriamente las condiciones de trabajo históricas del sector, también encontraron sus límites en las contradicciones inherentes a esta configuración productiva, que implementa un conjunto de dispositivos y normativas según las concepciones más modernas sobre seguridad, en un contexto marcado por la precarización de las relaciones laborales y por formas de gestión de la mano de obra con fuertes improntas tradicionales. Las políticas de gestión del riesgo de las empresas forestales muestran una configuración específica, que se distancia de las realidades encontradas en anteriores investigaciones en sectores industriales y de servicios, pero que también tiene características que las diferencian de los contextos laborales del mundo rural uruguayo.

\section{BIBLIOGRAFÍA}

Alamberti, R. (2012). Las claves de un enfoque sistémico eficaz en la gestión de riesgo. In Libro de actas, Safetygranada. Los factores humanos de la seguridad operacional en los sistemas complejos de alto riesgo (pp. 113-156). Granada : Actas de Congreso.

Argyris, C. (1995). Savoir pour agir. Surmonter les obstacles à l'apprentissage organisationnel. París : Inter Editions.

Beck, U. (1998). La sociedad del riesgo. Madrid : Editorial PAIDOS.

Beltrán, M. (1992). Cinco vías de acceso a la realidad social. In M. García Ferrando, J. Ibáñez, \& F. Alvira, (Comp.), El análisis de la realidad social. Métodos y técnicas de investigación (pp. 7-41). Madrid : Ed. Alianza Universidad Textos.

Boissières, I. (2007). Robustez organizacional y gestión del riesgo. In J. Walter, \& F. Pucci (Eds.), La gestión del riesgo y las crisis. Personas, culturas organizacionales e instituciones (pp. 155-188). Buenos Aires : Editorial El Ateneo, Universidad San Andrés.

Bourrier, M. (2001). La fiabilité est une question d'organisation. In M. Bourrier (Sous la direction de...), Organiser la fiabilité (pp. 9-38). París : L’Harmattan.

De la Garza Toledo, E. (2010). Hacia un concepto ampliado de trabajo. Del concepto clásico al no clásico. Ciudad de México : Editorial Anthropos, UNAM.

García Ferrando, M., \& Sanmartin, R. (1992). La observación científica y la obtención de datos sociológicos. In M. García Ferrando, J. Ibáñez, \& F. Alvira, (Comp.), El análisis de la realidad social. Métodos y técnicas de investigación (pp113-140). Madrid : Ed. Alianza Universidad Textos.

Giddens, A. (1995). La constitución de la sociedad. Buenos Aires : Amorrortu. 
Ibáñez, J. (1992) Perspectivas de la investigación social. In M. García Ferrando, J. Ibáñez, \& F. Alvira, (Comp.), El análisis de la realidad social. Métodos y técnicas de investigación (pp. 57-98).

Madrid : Ed. Alianza Universidad Textos.

Karpik, L. (1996). Dispositifs de confiance et engagements crédibles. Sociologie du Travail, 38(4), 527-550.

Koenig, G. (1994). Introduction : repérage des lieux [Dossier "L'apprentissage organisationnel : Pratiques et theories]. Revue Française de Gestion, 97, 76-83.

La Porte, T. (2001). Fiabilité et légitimité soutenable. In M. Bourrier (Sous la direction de...), Organiser la fiabilité (pp. 71-105). París : L'Harmattan.

Luhmann, N. (1992). Sociología del riesgo. Ciudad de México : Universidad Iberoamericana.

Novick, M., Yoguel, G., Catalano, A., \& Albornoz, F. (2002). Adaptación de modelos productivos en países emergentes. El caso de la industria automotriz en la Argentina. Buenos Aires : LITTEC, UNGS.

Orti, A. (1992) La apertura y el enfoque cualitativo o estructural : la entrevista abierta semidirectiva y la discusión de grupo. In M. García Ferrando, J. Ibáñez, \& F. Alvira, (Comp.), El análisis de la realidad social. Métodos y técnicas de investigación (pp. 153-185). Madrid : Ed. Alianza Universidad Textos.

Perrow, Ch. (1984). Normal accidents. New York: Basic Books.

Pucci, F. (2004). Aprendizaje organizacional para la gestión del riesgo. Montevideo : CINTERFOR, OIT.

Riella, A., \& Ramírez, J. (2007). Población rural y forestación : el estudio de la dinámica poblacional en los territorios forestales del Uruguay. Comunicación presentada a la $6^{a}$ Reunión Anual de Investigadores del Departamento de Sociología, Facultad de Ciencias Sociales, UDELAR, Montevideo.

Simard, M. (1998). Cultura y gestión de la seguridad. In Enciclopedia de Salud y Seguridad en el Trabajo (59.4- 59.8). Génova : OIT.

Vaughan, D. (2001). La normalisation de la déviance : une approche d'action située. In M. Bourrier (Sous la direction de...), Organiser la fiabilité (pp. 106-125). París : L’Harmattan.

Veltz, P., \& Zarifian, P. (1993). Vers de nouveaux modèles d'organisation ? Sociologie du Travail, 1(93), 3-25.

\section{RESÚMENES}

Este trabajo se propone analizar las políticas de gestión del riesgo en tres empresas forestales uruguayas de madera y pasta de papel, de alta dotación de capital y tecnologías modernas, cuyos procesos de gestión se modernizaron, pero que mantienen las modalidades de producción y de contratación tradicionales en el medio rural uruguayo. Las conclusiones son que la tercerización de los procesos de trabajo y la contratación por productividad, reproducen condiciones de trabajo en las cuales descentralización de responsabilidades, la carga física de la jornada y la competencia por lograr los rendimientos exigidos, establecen fuertes contradicciones con las modernas políticas de gestión del riesgo implementadas.

Este trabalho pretende explorar três empresas florestais uruguaias de madeira e pasta de papel, com alto investimento de capital, cujos processos de trabalho foram modernizados e alcançaram uma relativa melhoria das condições de trabalho que existiam anteriormente, mas que mantêm modos de recrutamento e de produção em forte contradição com as mudanças. A terceirização de processos de trabalho é associado com a presença de formas de contratação por produtividade 
e condições de trabalho em que a carga física e a concorrência para conseguir o performances exigidas pelas empresas, agravam as dificuldades no estabelecimento de uma política eficiente de gestão de risco.

Ce travail a pour objectif l'analyse des politiques de gestion du risque dans trois entreprises de production de bois en Uruguay. Depuis les années 90, la production de bois de l'Uruguay a subi une forte croissance, suite à l'implantation, en différentes régions du pays, de nombreuses entreprises étrangères et nationales qui ont procédé à d'importantes inversions en technologie et équipements. Par contre, les formes classiques de travail dans les zones rurales, marquées par la sous-traitance, la forte charge physique et la concurrence autour de la productivité individuelle, entrent en contradiction avec les politiques de modernisation de la gestion de la force de travail dans les nouvelles entreprises de production de bois.

The paper examines three Uruguayan forest companies of wood and paper pulp sector with high capital investment, whose work processes have been modernized and achieved a relative improvement in working conditions. However, it still remains patterns of production and recruitment that come in strong contradiction with those changes. The outsourcing of work processes is associated with the presence of forms of hiring by productivity, which reproduces the conditions in which the physical burden of the day and the competition to achieve the performances required by the companies, exacerbate the difficulties in establishing efficient politics of risk management.

\section{ÍNDICE}

Keywords: management risks, forest industry, Uruguay

Palavras-chave: gerenciamiento riscos, setor florestal, Uruguai

Mots-clés: gestion risque, industrie production de bois, Uruguay

Palabras claves: gestión riesgo, industria forestal, Uruguay

\section{AUTORES}

\section{FRANCISCO PUCCI}

Profesor Titular del Departamento de Sociología de la Facultad de Ciencias Sociales, UDELAR. Constituyente 1502, piso 5. CP 11.200 Montevideo, Uruguay

pucci@fcs.edu.uy

\section{SOLEDAD NIÓN}

Profesora Asistente del Departamento de Sociología de la Facultad de Ciencias Sociales, UDELAR. Constituyente 1502, piso 5. CP 11.200 Montevideo, Uruguay msnion@gmail.com

\section{FIORELLA CIAPESSONI}

Profesora Ayudante del Departamento de Sociología de la Facultad de Ciencias Sociales, UDELAR. Constituyente 1502, piso 5. CP 11.200 Montevideo, Uruguay fciape@gmail.com 